\title{
Endoscopic anatomy of the cerebellopontine angle: a study in cadaver brains
}

\section{Patrick Chaynes, M.D., Olivier Deguine, M.D., Jacques Moscovici, M.D., Bernard Fraysse, M.D., Jean Bécue, M.D., and Yves Lazorthes, M.D.}

Laboratoire d'Anatomie, Service de Neurochirurgie, Service d'Otologie, Faculté de Médecine Toulouse-Rangueil, France

The increasing trend toward performing minimally invasive neurosurgery may benefit from recent progress in using neuroendoscopic techniques to reduce trauma in patients who have undergone operations. Arterial and venous vessels, especially loops, may compress the central segment and cause hyperactive dysfunction of the nerves. Relationships of the anterior inferior cerebellar artery to the facial and vestibulocochlear nerves and the anterior inferior, and superior cerebellar arteries to the trigeminal nerve were studied. The authors report findings from an endoscopic study performed in cadaver heads via the retrosigmoid and retrolabyrinthine approaches. Arteries and veins were colored by injection of red and blue silicon rubber. The cerebellopontine angle (CPA) was examined using 2.7-mm and 4-mm-diameter rigid endoscopes at viewing angles of $0 \check{s}, 30 \check{s}$, and 70š. Well-known structures could be identified endoscopically without prior dissection, and the entire CPA could be explored. However, with a retrosigmoid or a retrolabyrinthine approach, the cerebellum had to be retracted to some extent to view the CPA. Moreover, wide dural exposure was required to maneuver the endoscope freely in the CPA. Use of the rigid fiberoptic endoscope is not yet superior to standard surgical techniques for approaching and exploring the CPA.

Key Words * neuroendoscopy * cerebellopontine angle * microsurgery * anatomical study

The cerebellopontine angle (CPA) is one of the most important sites for functional neurosurgery. A variety of operations may be performed in this area, the most common of which is microvascular decompression of the cranial nerve roots and section of the vestibular nerve. The indications for this procedure include trigeminal neuralgia, hemifacial spasm, tinnitus, vertigo and glossopharyngeal neuralgia caused by vascular compression of the central segment of the fifth, seventh, eighth, and ninth cranial nerves.[10] The major approaches to the region are suboccipital craniectomy and its retrosigmoid variation. To minimize side effects of potentially traumatic surgery, a variety of keyhole approaches have been developed. Another technique could involve the use of a device such as a neuroendoscope. The continued development of these techniques now enables surgeons to routinely reach the lateral and third ventricle to treat hydrocephalus or to obtain biopsy samples of intraventricular tumors.[6,17] Endoscopic 
approaches to the CPA are less well documented in the literature. Because this region of arachnoid cisterns provides a natural pathway to the major intracranial vessels and cranial nerves, neurovascular decompression could be performed via an endoscopic approach to the CPA. Knowledge of the topography and microanatomy of the relationships between nerves and vessels is fundamental for diagnosis and treatment, particularly in functional neurosurgery. These considerations prompted us to evaluate the endoscope for the exploration, diagnosis, and treatment of lesions, especially functional, of the CPA. The anatomy and the relationships of the surrounding structures of the CPA were studied through endoscopic views.

\section{MATERIALS AND METHODS}

Both CPAs in four cadaver brains were studied endoscopically. Each fresh specimen was injected with colored silicone rubber to aid identification of the vascular structures. The study was performed after brain specimens were fixed in $10 \%$ formalin for a short duration to provide more consistency to the brain. Two rigid endoscopes with outer diameters of $2.7 \mathrm{~mm}$ and $4 \mathrm{~mm}$ with variable-angled $(0,30$, and $70^{\circ}$ ) lens scopes (Wolf, Knittlingen; Storz, Tuttlingen, Germany) were used. In all cases, a xenon light source provided illumination. The endoscopes were connected to a camera system, and duplicate pictures were transmitted to the video display unit. The endoscopic approach and anatomical details were observed and documented with videotapes and slides.

\section{Surgical Procedure}

We performed eight craniectomies: six for the retrosigmoid (extending from between $10 \mathrm{~mm}$ and $25 \mathrm{~mm}$ ) and two for the retrolabyrinthine (extending approximately $15 \mathrm{~mm}$ ) approaches on both sides. For the "keyhole" approach to microvascular decompression, the most critical portion is the precise positioning of the burr hole. The sigmoid sinus is the anterior limit of dissection, and it is exposed and retracted anteriorly. The transverse sinus lies along the axis of a line connecting the root of the zygoma with the external occipital protuberance. This line corresponds to the superior nuchal line, and in the retrosigmoid approach, the superior nuchal line marks the superior limit of the craniotomy. The back side of the body of the mastoid approximates the anterior craniotomy margin, correlating closely with the posterior margin of the descending segment of the sigmoid sinus. The burr hole is placed just inferior to the superior nuchal line, with the anterior portion of the hole over the back side of the body of the mastoid at the junction of the mastoid process and the transverse sinus.

After craniectomy, the dura mater was incised, and the whole cisterns of the posterior cranial fossa were explored. The ease of exploring the CPA was then evaluated in relation to the size of the craniectomy. The location and the relationships of the nerves and vessels were also observed.

\section{RESULTS}

The size of the craniectomy extended from small burr hole to wide exposure. When the size of the burr hole was smaller than $10 \mathrm{~mm}$, the endoscope was maneuvered with difficulty, and the whole CPA could not be explored; however, the endoscope could be maneuvered freely within the CPA when burr holes were larger than $15 \mathrm{~mm}$. We could explore nearly all the CPA structures and even the ventral surface of the pons and the clivus in most brains (Fig. 1), and the anatomical structures and their relationships were visualized in considerable detail. 


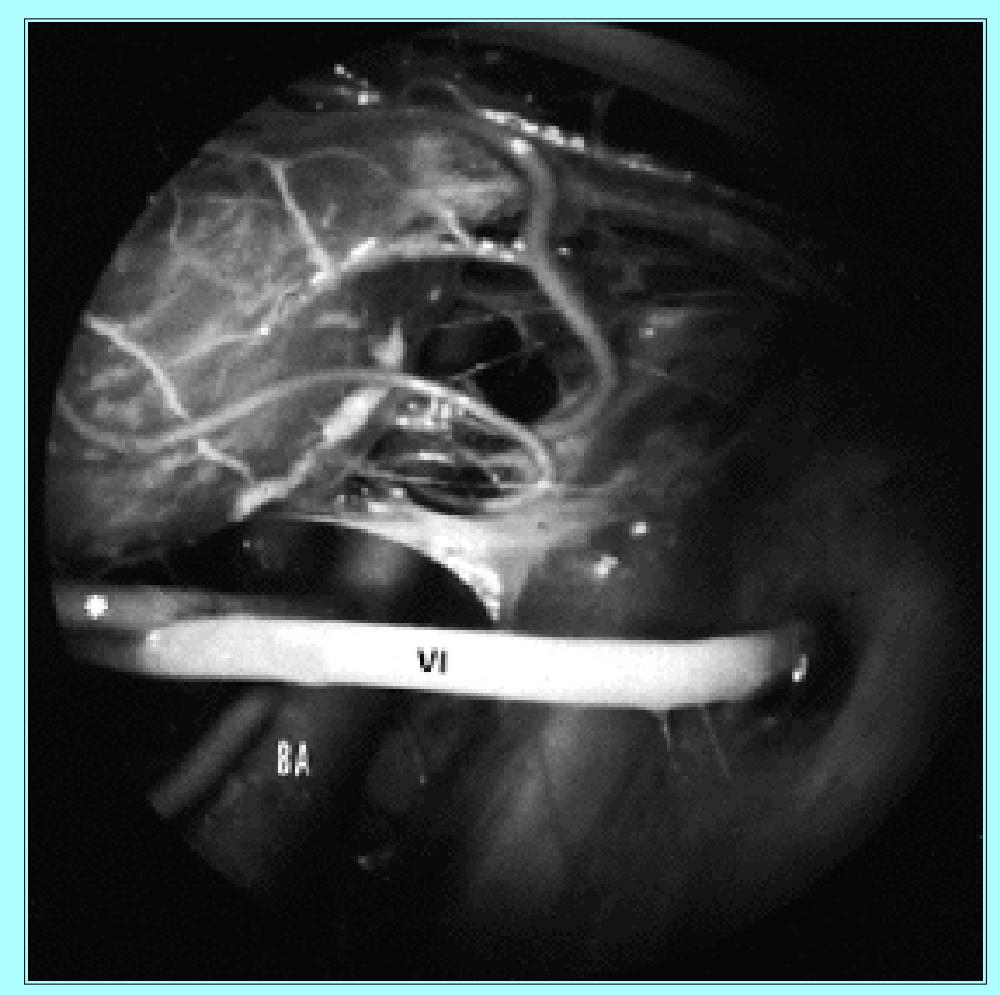

Fig. 1. Endoscopically obtained image showing the ventral surface of the pons and clivus. The abducens nerve (asterisk) arises from the pontomedullary sulcus and penetrates the dura mater of the clivus passing into Dorello's canal (arrow). The BA is located medially and gives rise to the anterior inferior cerebellar artery (asterisk). Note the perforating arteries of the $\mathrm{BA}$ to the pons. $\mathrm{BA}=$ basilar artery; $\mathrm{VI}=$ sixth cranial nerve.

The diameter of the burr holes or the craniectomies were 15, 20, and $25 \mathrm{~mm}$ on each of the sides for the retrosigmoid approaches and $15 \mathrm{~mm}$ for the retrolabyrinthine approaches. In some cases, there was atrophy of the cerebellum, which was retracted to explore the whole CPA, but it was relatively easy to navigate within the cisterns of the posterior fossa. The arachnoid membrane was well conserved, and its trabeculae could be well visualized (Fig. 2). 


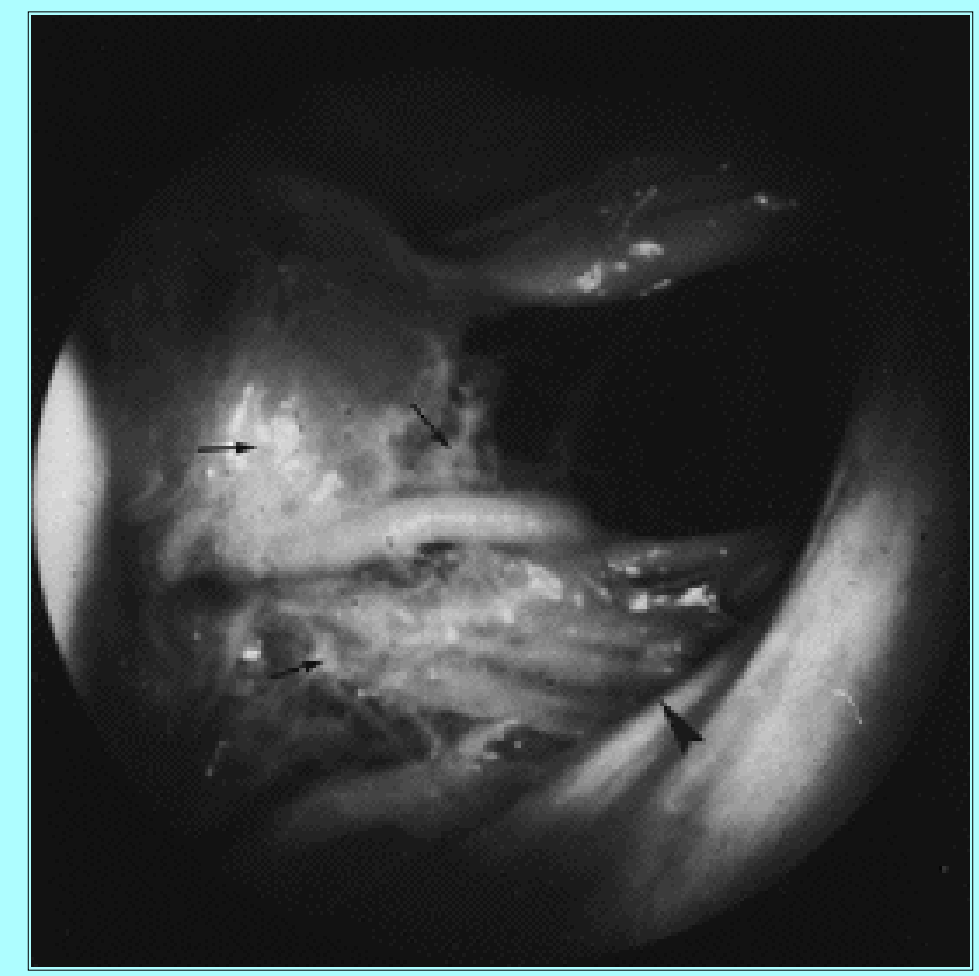

Fig. 2. Endoscopically obtained image showing the caudal portion of the right CPA. The arachnoid membranes and trabeculae (arrows) of the CPA are well conserved and seen covering the lower cranial nerves reaching the jugular foramen (arrowhead). The membrane needs to be torn to explore the whole cisterns of the posterior fossa.

Their aspect depended on the specimen being studied. The membranes were better preserved when the procedure was performed within 4 days after death. The arachnoid membranes were sometimes pierced, torn, and retracted around the vessels and nerves and this configuration facilitated exploration within the CPA. When the arachnoid was not pierced and covered the vessels and cranial nerves, it was difficult to pass the endoscope through it without the aid of another instrument. Even if it was not possible to explore the CPA with a 10-mm burr hole, the superior part could be visualized depending on the location of the burr hole. There were no marked differences in endoscopic visualization when a burr-hole diameter was larger than $15 \mathrm{~mm}$. This approach was identical to that required for standard keyhole retromastoidal microsurgery. However, the various viewing angles facilitated exploration of areas not directly illuminated by the microscope.

The CPA was bordered anteriorly by the dura covering the posterior surface of the petrosal bone and posteriorly by the anterior surface of the cerebellum, superiorly by the pons and the middle cerebellar peduncle, inferiorly by the biventral lobule, and medially by the inferior olive. It was constituted by the cerebellopontine and cerebellomedullary cisterns, which were separated by the lateral pontomedullary membrane. Medially, the cerebellopontine cistern was separated from the prepontine cistern by the anterior pontine membrane, and the cerebellomedullary cistern was separated by trabeculae in front of the lower cranial nerves. The nerves formed three distinct bundles, craniocaudally and mediolaterally dividing the CPA into three compartments: cranial, medial, and caudal. Each compartment was crossed by a major artery and a group of cranial nerves. The cranial compartment was crossed by the trigeminal nerve in a sagittal direction accompanying the anterior inferior and superior cerebellar arteries; the abducens nerve coursed medially (Fig. 3). 


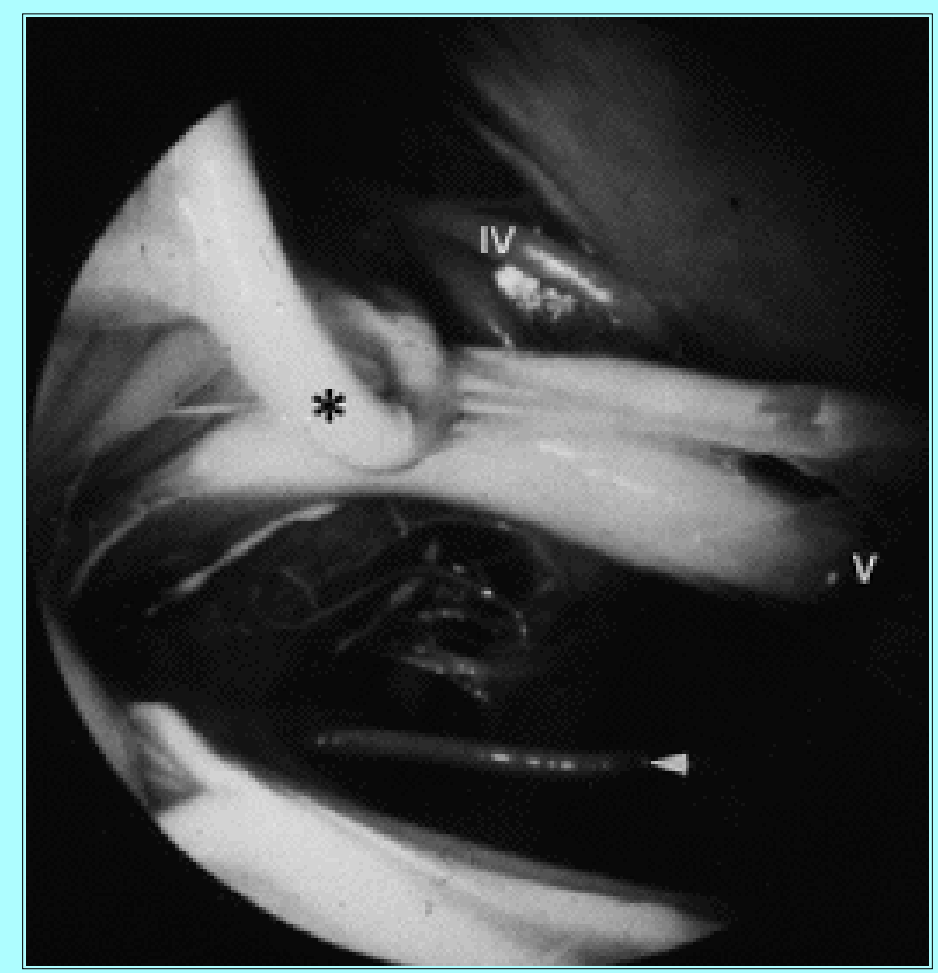

Fig. 3. Endoscopically obtained image of the cranial portion of the right CPA. The trigeminal nerve courses sagittally to the trigeminal cave (Meckel's cave). The anterior superior cerebellar artery (asterisk) is close to the superior part of the nerve, whereas the inferior part is in contact with a pontic vein. Medially, the abducens nerve courses through the cistern to Dorello's canal (arrowhead). The trochlear nerve may be seen around the cerebral peduncle. $\mathrm{V}=$ trigeminal nerve; $\mathrm{IV}=$ trochlear nerve.

The trigeminal nerve stemmed from the lateral part of the pons, whereas the abducens nerve arose at the level of the pontomedullary sulcus and ascended just lateral to the basilar artery. The facial, intermedius and vestibulocochlear nerves coursed obliquely upward and laterally, with the posterior inferior and anterior inferior cerebellar arteries, within the medium compartment to reach the porus acusticus (Fig. 4). 


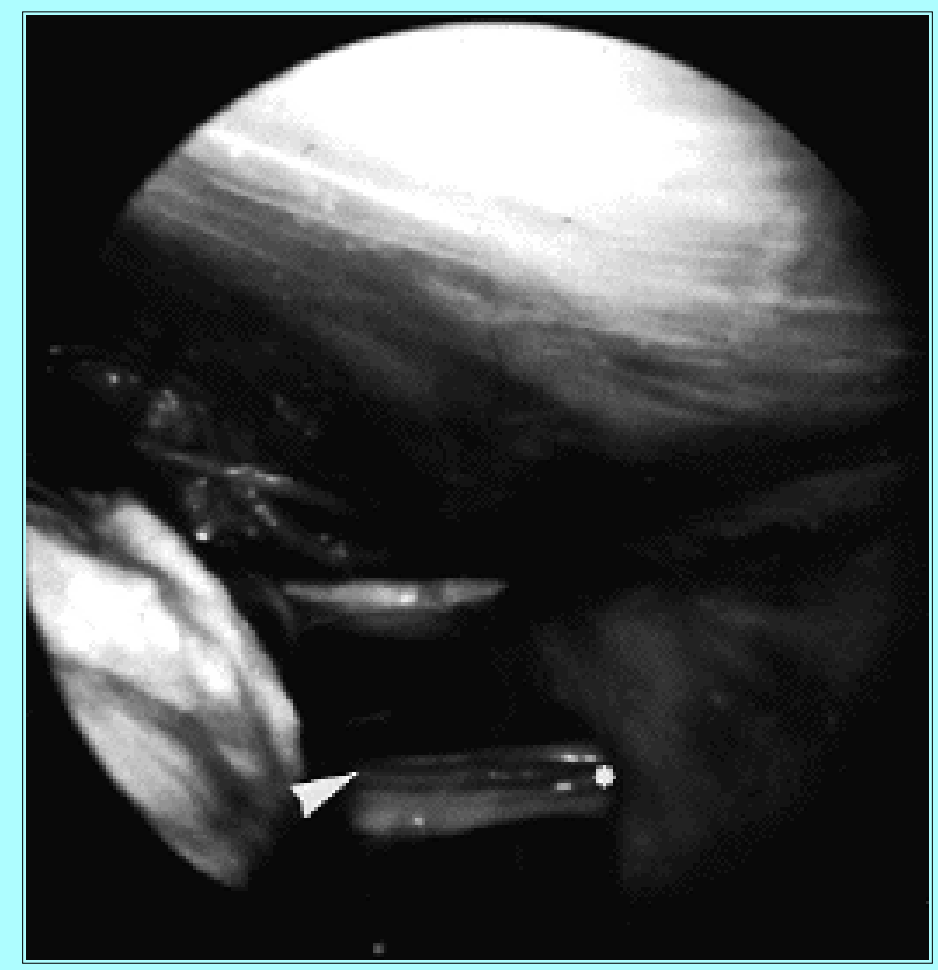

Fig. 4. Endoscopically obtained image of the medial compartment of the right CPA showing the acousticofacial bundle, which courses to the internal porus (asterisk). The internal auditory artery (arrowhead) courses in a sulcus between the facial nerve anteriorly and the vestibulocochlear nerve posteriorly.

The caudal compartment was crossed by the posterior inferior cerebellar artery and the lower cranial nerves (glossopharyngeal, vagus, and accessory nerves) slanting down, ventrally and laterally, to reach the jugular foramen (Fig. 5). This compartment was separated from the caudal compartment by the lateral pontomedullary membrane, which crossed the subarachnoid space between the vestibulocochlear and glossopharyngeal nerves in front of the flocculus.

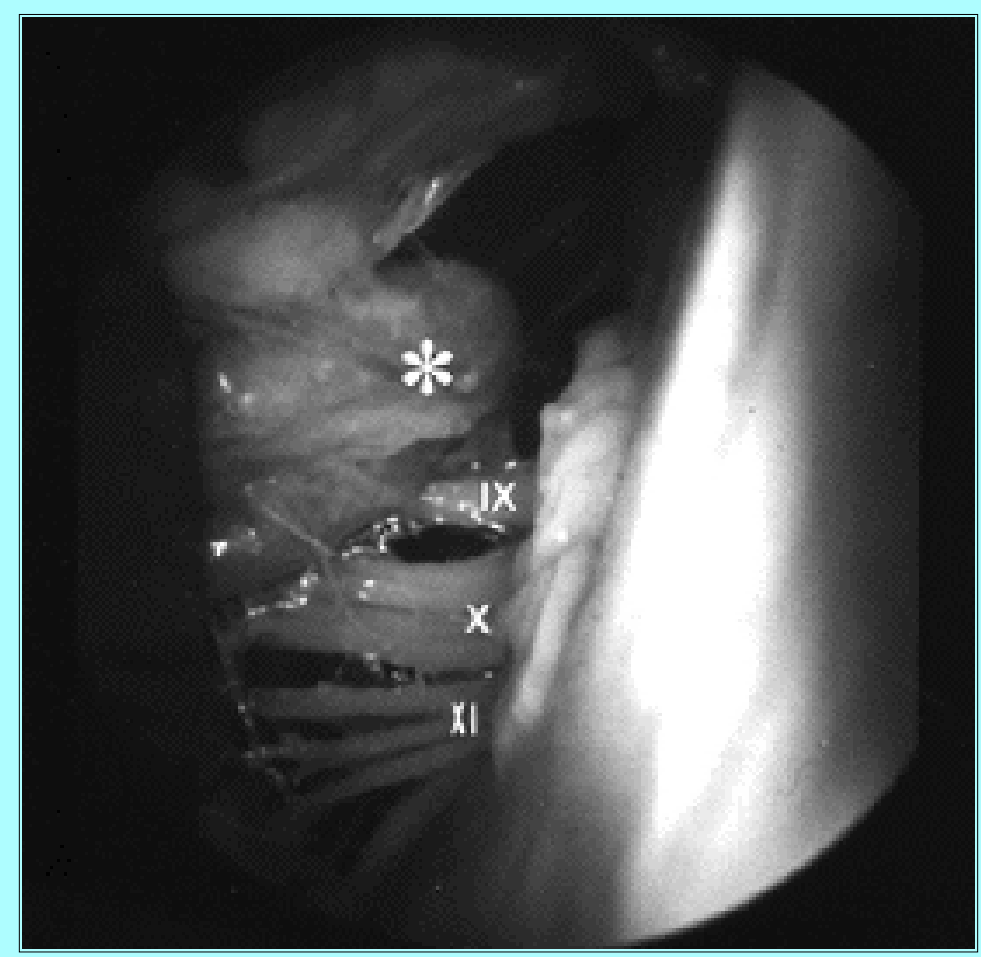


Fig. 5. Endoscopically obtained image showing the caudal compartment of the right CPA. The lower cranial nerves are accompanied by a loop of the posterior inferior cerebellar artery (asterisk). IX =glossopharyngeal; $\mathrm{X}=$ vagus; $\mathrm{XI}=$ accessory nerve.

\section{DISCUSSION}

Endoscopic techniques are becoming more widely used,[5,8,11] and endoscopically guided operations in the supratentorial ventricular system are well documented and now regarded by many authors as established neurosurgical techniques.[11] Most current clinical applications of neuroendoscopy concern the ventricular system.[2,3,6,14] The few reports on its use in the basal cistern have mostly involved cadaveric studies. $[7,13,15]$ However, it would be of considerable interest to explore the whole CPA endoscopically, because it would enable examination of many internal sites with minimal trauma.[7,11] It was first described in a translabyrinthine approach by Prott,[16] but we are not aware that it has been used surgically.

We studied the CPA in cadaver brains in an attempt to simulate the operative procedure for exploration and microvascular decompression. This model provides a good overview for endoscopic navigation and has been widely used to determine the feasibility of viewing the anatomical structures in the brain and basal cisterns through rigid or flexible endoscopes.[7,11,13,14,17] However, the cerebellar atrophy in the cadaver brain enlarges the basal cisterns, which facilitates neuroendoscopic navigation. The basal cisterns in cadaver brains were larger than those found in vivo. With some retraction of the cerebellum, we could explore all the basal cisterns and inspect the arachnoid membranes and trabeculae. The complex appearance reported by Matula, et al.,[11] could be accounted for by retraction of the arachnoid around the vessels and nerves.

Arterial and venous vessels, especially loops, may compress the central segment, leading to hyperactive dysfunction of the nerves. Jannetta[10] has reported that lesions from vessels involving the central segment of the fifth, seventh, eighth, and ninth cranial nerves that cause trigeminal neuralgia, hemifacial spasm, tinnitus, vertigo, and glossopharyngeal neuralgia can be treated surgically by ablation of the neurovascular conflict. It is routinely performed under magnification through a retromastoid approach, a variation of the suboccipital approach. For the keyhole approach to microvascular decompression, the most critical aspect is the precise positioning of the burr hole.[4,7] The retrosigmoid approach provides a more lateral view of the CPA than does the classic suboccipital approach.[12] The retromastoid approach provides essentially a tangential view of the 8th cranial nerve, 70 to 90 š to the long axis of the nerve.[12] This angle of view could be improved through a retrolabyrinthine approach, giving an angle of view from approximately 30 to 70 š posterior to the 8th cranial nerve. It was introduced by Hitselberger and Pulec,[9] who used it for access to the fifth cranial nerve and to perform microvascular decompression of the facial nerve root,[1] and by Silverstein, et al.,[18] for selective section of the vestibular nerve in the posterior cerebral fossa.

Some authors claim that endoscopic techniques are superior to open craniotomy for demonstration of in vivo relationships.[11] The microscope, however, has distinct limitations: the operator can only visualize structures directly illuminated by the microscope and cannot see around objects or down narrow, tortuous pathways. Access to the site of disease may thus require wide exposure and retraction of adjacent structures.[13] The endoscopes used thus far do not possess an internal canal and, therefore, do not allow passage of other instruments, making dissection of the arachnoid rather difficult. The small vessels are not readily dissected from the arachnoid membrane, and it is not easy to staunch bleeding induced by 
rupture of such vessels. Another major drawback of endoscopes is their poor depth perception compared with binocular microscopy. However, a variety of angles are available in rigid endoscopes, although with the flexible endoscopes, illumination is poorer and the image less sharp.[13] At the present time, rigid fiberoptic endoscopy has no advantages over standard microsurgery for the surgical approach and exploration of the CPA. The endoscope is best used in conjunction with the microscope, thus offering more complete exploration of the CPA. For example, the remaining tumor located within the internal auditory canal is not easily seen using the microscope, whereas it can be well seen using an endoscope with variable-angled lens and scopes. During exploration of the CPA, the ventral surface of the pons and clivus can be easily seen through a retromastoid approach when using 30 š and 70š lens scopes. Endoscopy is thus a valuable adjunct to conventional surgical techniques.

\section{References}

1. Brackman DE, Hitselberger WE: Retrolabyrinthine approach: technique and newer indications. Laryngoscope 88:286-297, 1978

2. Caemaert J, Abdullah J: Diagnostic and therapeutic stereotactic cerebral endoscopy. Acta Neurochir 124:11-30, 1993

3. Dandy WE: Cerebral ventriculoscopy. Johns Hopkins Hosp Bull 33:189, 1922

4. Day JD, Kellogg JX, Tschabitscher M, et al: Surface and superficial surgical anatomy of the posterolateral cranial base: significance for surgical planning and approach. Neurosurgery 38:1079-1084, 1996

5. Decq P, Yepes C, Anno Y, et al: L'endoscopie neurochirurgicale. Indications diagnostiques et thérapeutiques. Neurochirurgie 40:313-321, 1994

6. Drake JM: Ventriculostomy for treatment of hydrocephalus. Neurosurg Clin North Am 4:657-666, 1993

7. Fukushima T: Endoscopy of Meckel's cave, cisterna magna, and cerebellopontine angle. Technical note. J Neurosurg 48:302-306, 1978

8. Griffith HB: Endoneurosurgery: endoscopic intracranial neurosurgery. Adv Tech Stand Neurosurg 14:2-24, 1986

9. Hitselberger WE, Pulec JL: Trigeminal nerve (posterior root) retrolabyrinthine selective section. Operative procedure for intractable pain. Arch Otolaryngol 96:412-415, 1972

10. Jannetta PJ: Arterial compression of the trigeminal nerve at the pons in patients with trigeminal neuralgia. J Neurosurg 26:159-162, 1967

11. Matula C, Tschabitscher M, Kitz K, et al: Neuroanatomical details under endoscopic view - relevant for radiosurgery? Acta Neurochir Suppl 63:1-4, 1995

12. Monsell EM, McElveen JT Jr, Hitselberger WE, et al: Surgical approaches to the human cochlear nuclear complex. Am J Otol 8:450-455, 1987

13. O'Donoghue GM, O'Flynn P: Endoscopic anatomy of the cerebellopontine angle. Am J Otology 
14:122-125, 1993

14. Oka K, Go Y, Kin Y, et al: An observation of the third ventricle under flexible fiberoptic ventriculoscope: normal structure. Surg Neurol 40:273-277, 1993

15. Perneczky A, Tschabitscher M, Resch KDM: Endoscopic Anatomy for Neurosurgery. New York: Stuttgart-Thieme, 1993

16. Prott W: [Cisternoscopy of the cerebellopontine angle.] HNO 22:337-341, 1974 (Ger)

17. Riegel T, Hellwig D, Bauer BL, et al: Endoscopic anatomy of the third ventricle. Acta Neurochir Suppl 61:54-56, 1994

18. Silverstein H, Norell H, McDaniel AB, et al: Retrolabyrinthine vestibular neurectomy, in Wiet RJ, Causse JB (eds): Complications in Otolaryngology-Head and Neck Surgery. Vol 1: Ear and Skull Base. Toronto: BC Decker, 1986

Manuscript received July 14, 1998.

Accepted in final form August 14, 1998.

Address reprint requests to: Patrick Chaynes, M.D., Service de Neurochirurgie, CHU Rangueil, 1 avenue Jean Poulhès, F-31054 Toulouse Cedex, France. 